\title{
Identification of Key Nodes in Power Grid Based on Node Flow
}

\author{
SUN Zhiyuan ${ }^{1, a, *}$, SUN Yan ${ }^{2, b}$ and LIANG Shuiying ${ }^{1, \mathrm{c}}$ \\ ${ }^{1}$ Guangxi Power Grid Electric Power Research Institute, Nanning, China \\ ${ }^{2}$ Guangxi power grid dispatching control center, Nanning, China \\ ${ }^{3}$ Guangxi Power Grid Electric Power Research Institute,, Nanning, China \\ asun_zy.sy@gx.csg.cn, ${ }^{b}$ sun_y.dd@gx.csg.cn, ${ }^{c}$ liang_sy.sy@gx.csg.cn
}

Keywords: key node; power flow transfer; AC/DC hybrid power grid; node flow; cascading failures

\begin{abstract}
Some key nodes in the power grid may have an important role in the cascading failures. In order to improve the accuracy of key node identification and prevent the occurrence of cascading failures more effectively, this paper proposes a key node identification method based on the node flow. This method uses the 2 factors, which are the change of the network capacity after failure and the electrical coupling strength between nodes, to quantitatively characterize the important position of a node in the transmission of cascading failures. The proposed method can quantitatively characterize the influence of the power flow caused by the interruption of a certain AC/DC line to the surrounding line. It can quickly and accurately identify the weak nodes in the power grid and judge the important role that they played in the evolution of AC/DC power grid, which are of great significance to prevent the evolution from cascading failure system to the critical state. The simulation results of a regional power grid system show that the proposed method can be used to identify the key nodes in the power grid more effectively and accurately.
\end{abstract}

\section{Introduction}

With the increasing size of the power system, the power system of our country has gradually become a hybrid complex large AC/DC power grid, and the complexity of its form also increases. As a result of this AC/DC parallel operation, complex structure and tight connection, making the safe and stable operation of the power grid situation becomes more complicated. In recent years, there have been frequent blackouts at home and abroad, resulting in serious economic losses and poor social impact ${ }^{[1-4]}$. After many experts and scholars at home and abroad researched for several years, it has been found that these blackouts in power system are closely related to some of the key nodes of the power grid, and these key nodes play a very important role in the safe and stable operation of the power system. The failure of these key nodes can easily lead to cascading failure, and then lead to power grid instability, collapse. Therefore, how to identify the key nodes in the power grid fast and accurately according to the actual situation is very important. ${ }^{[5-6]}$ Accurate and rapid identification of these key nodes in the power system could make us targeted to take 
protective control strategies, to improve the safe and stable operation of the power system and to prevent the occurrence of power system cascading failure caused by a major blackout.

At present, some researches on the identification of key nodes in power system have just made some achievements. According to the existing identification of key nodes, these studies can be divided into two categories by different modelling direction.

The first type of researches from the view of complex networks, taking the topology of the power grid into account, studies the topological parameters from the structural point of the power grid, using small world network model ${ }^{[7]}$ and scale network model ${ }^{[8]}$ and other complex network theory to identify key nodes in the power grid. In literature [9-10], the importance of lines and nodes is identified by the betweenness of lines and nodes, and then the evolution of power system cascading fault is analyzed with the betweenness. In literature [11], it is pointed out that there are a few nodes in the power system, and their ordinal indexes are much larger than those of other nodes. Faults of such nodes can easily lead to the occurrence of power grid system cascading fault. In literature [12], considering the topological structure of the power system network and the electrical characteristics of the power grid, the weighted betweenness of lines is used to identify the key aspects of power system that have a critical effect on the system due to its own topological location.

The second type of research is based on the actual physical properties of the power grid. In literature [13], the electrical distance between the power grid nodes is improved to the voltage coupling strength and the probability of the line, which makes up for the lack of sufficient attention to the relationship between the model and the topology. In literature [14], based on the theory of network maximum flow and complex network, the transmission contribution is defined to characterize the carrying capacity of key nodes to the power transmission function of power grid. The literature [15] proposed the line betweenness of power flow, which overcomes the drawbacks that the previous power is transmitted along the shortest path of node and have no directivity. In literature [16], the power grid topology, power transmission relationship and system state are integrated to make quantitative description.

Previous research has some reference significance, but whether from the complex network theory topology analysis direction, or from the actual physical properties of the power grid direction, are not considered comprehensive. In the $\mathrm{AC} / \mathrm{DC}$ power grid, the degree of the node can not characterize the key degree of the nodes in the power grid. If this node is a DC line converter station, the failure of this node will lead to DC line locking, which has an important impact on the transmission of cascading failures. At the same time, the role of the line in the power transmission is closely related to its power, but is not necessarily linked with the degree of the node size. Therefore, a new indicator is needed to reflect the impact of a node failure on the power grid in AC/DC power networks.

In conclusion, this paper defines the concept of node flow, which can quantitatively characterize the reduction of power capacity before and after a node in the power grid blackout. When characterizing the key degree of nodes in the power grid, compared with the complex network theory node degree and the effective performance model in the network efficiency and other methods, this definition can overcome the drawbacks that the above method can be only related to the power grid topology or trend distribution. In this paper, the important position of a node in the network cascading failure propagation is quantitatively represented by the change of the network capacity and the change of topology structure after the node failure. The key node circulation importance model proposed in this paper can accurately identify key nodes in the AC/DC hybrid power system, and the validity and accuracy of the model and algorithm are verified by simulating a regional power grid. 


\section{The conception of node flow}

The modern power system consists of a large number of transmission lines, generators, circuit breakers, transformers and loads. In this paper, the power grid is abstracted as an undirected weighted network, the power station, substation and load are abstracted as a node, power lines are abstracted as edge.

In terms of the power grid, the simple complex network theory ignores the actual physical characteristics of power grid (such as resistance, reactance, flow characteristics) and effective constraint conditions (load size, generator output limit, etc.), which cannot capture the running characteristics of power grid. Therefore, it is very important to select the appropriate topological weight to characterize the importance of power grid components. Because the impedance difference of each line in the power grid is large, it affects the distribution of the power flow to a great extent. Therefore, it is necessary to introduce the line impedance value into the power grid model to construct a weighted power grid model. In addition, considering the resistance of high voltage transmission lines is often far less than the branch reactance, in view of this, this paper ignores the line resistance, only obtaining the difference from the transmission line reactance, and proposes a weighted network model through the line reactance. Define power grid node importance degree first as follows:

$$
A_{E}=\left[\begin{array}{cccc}
E_{1} & \omega_{12} E_{2} / K^{2} Z_{12, \text { equ }} & \cdots & \omega_{1 n} E_{n} / K^{2} Z_{1 n} \text {, equ } \\
\omega_{21} E_{1} / K^{2} Z_{21, \text { equ }} & E_{2} & \cdots & \omega_{2 n} E_{n} / K^{2} Z_{2 n} \text {, equ } \\
\vdots & \vdots & \cdots & \vdots \\
\omega_{n 1} E_{1} / K^{2} Z_{n 1, \text { equ }} & \omega_{n 2} E_{2} / K^{2} Z_{n 2} \text {, equ } & \cdots & E_{n}
\end{array}\right]
$$

In (1), $\mathrm{n}$ is the number of nodes in the power grid; $\mathrm{i}, \mathrm{j}$ is the grid node number, $\omega_{i, j}$ is the corresponding elements of the adjacency matrix of the power grid. when the node $\mathrm{i}$ and $\mathrm{j}$ connected, takes 1 , otherwise takes $0 ; \mathrm{K}$ is the network average Value, $Z_{i j, \text { equ }}$ is the electrical distance between the node $\mathrm{i}$ and the node $\mathrm{j}$, which can more accurately reflect the electrical coupling between the nodes; $Z_{i j, e q u}$ is numerically equal to the voltage $U_{g l}$ between nodes $g$ and 1 after injecting the unit current element from node g, as shown in (2).

$$
Z_{g l, e q u}=\frac{U_{g l}}{I_{g}}=U_{g l}
$$

According to the superposition principle, ${ }^{Z_{\text {gl, equ }}}$ can be expressed by the node impedance matrix elements, in which $Z_{g l}$ is the node impedance matrix G line L column elements. $E_{m}$ is the energy efficiency function for node $\mathrm{m}$, dimensionless, which is defined as follows:

$$
E_{m}=\frac{1}{n(n-1)} \sum_{i=1, i \neq m}^{n} \frac{1}{d_{m i}}
$$

$\operatorname{In}(4), d_{m i}$ is the shortest electrical distance between the node $\mathrm{m}$ and I. In this paper, all the power lines are reduced to non-directional power, the weight of the line is the line reactance value. The shortest electrical path in the power grid is the path with the smallest sum of the weight along all 
paths between two points. The shortest electrical distance is the electrical path with the smallest sum of the weight along the line ${ }^{[17]}$.

The main reason for the occurrence of the cascading failure is the trend transfer: while the system is under normal operating conditions, each component should carry a certain original load. If the system suffers from a factor that causes one or more of the components in the network to be overloaded, the current balance will be changed and the load will be reallocated on other components; If this part of the previous normal operation components cannot share the extra load, it will cause another load reallocation, causing the system to occur over-load fault, and ultimately lead to the occurrence of a large blackout event.

In the linear model of the power system, the power transmission distribution factors (PTDF) matrix can describe the contribution of each line in tidal current transmission. The matrix reflects the sensitivity of the power flow on the transmission line to a pair of load nodes and generator nodes. When a transmission line is disconnected, the larger the change of the network capacity, from the structural point of view, the more important the line to the normal operation of the power grid. However, PTDF is only determined by the network structure, which is the inherent property of transmission lines. After determining the grid structure of the power grid, the network capacity based on PTDF is also determined ${ }^{[14]}$.

Combined with the transmission capacity and PTDF, the power transmission capacity index is defined $L_{i j \in(g, l)}$ as the line weight:

$$
L_{i j \in(g, l)}=\frac{R_{i j}}{\left|R_{i j \in(g, l)}\right|}
$$

In (5), $R_{i j}$ is maximum power transmission capacity of line $\mathrm{i}, \mathrm{j}$.

The physical meaning of (5) is that the line power transmission capacity is related to the line transmission capacity and PTDF. The larger the line transmission capacity, the stronger the line power transmission capacity; the smaller the PTDF, the less sensitive the power transmission between the pair, the smaller the degree of contribution, the greater the capacity to tap and utilize the transmission capacity. Compared with the maximum active transmission capacity of the line, the line power transmission capability index more intuitively quantifies the contribution capacity of the power transmission between different nodes. It should be noted that, for the same line, for power transmission between the different generator load node pair, the line power transmission capacity is different, indicating the different carrying capacity of the line with different power transmission mode.

Combined with the characteristics of the maximum flow of the network and the actual operating characteristics of the power grid, the power node corresponds to the source point; the load node corresponds to the sink; the line corresponds to side; the actual direction of the trend corresponds to the direction of the corresponding side; the line power transmission capacity corresponds to the capacity of the side.

The contribution index of transmission line $\lambda_{i j \in(g, l)}$ based on single generator load node pair is defined as follows:

$$
\varphi_{i j \in(g, l)}=\sum_{p=1}^{N_{l}} L_{\min , p}
$$

In (6), $\mathrm{i}, \mathrm{j}$ is the line which can make contributions to the $(\mathrm{g}, \mathrm{l})$ node where PTDF is not 0 ; P is the power transfer path between the $(\mathrm{g}, 1)$ node pairs; $N_{l}$ is the total number of possible 
transmission paths between node pairs; $L_{\min , p}$ is the capacity value of the least power transmission line in the $\mathrm{P}$ transmission path

The index reflects the power transmission task of the line while the trend is transmitted between a single generator load node pair. The size of the index value reflects the depth of the line is used.

However, if there is a non-single generator load node pair in the power grid, a certain line may be utilized by different generator load node pairs. The number of generator load node pairs on the line reflects the breadth of the line usage.

From the point of view of the generator load node pairs to the power transmission, considering the cumulative effect, the global transmission contribution index $\varphi_{i j}$ is defined as

$$
\varphi_{i j}=\sum_{g \in G} \sum_{l \in L} \varphi_{i j \in(g, l)}
$$

In (7), G for the power node set; $L$ for the load node set. This indicator combines the depth and breadth of the line used in the transmission path between the different generator load node pairs. The breadth is the number of generator load node pairs that are utilized, and the depth is the sum of the amount of power transmission between generator load node pairs.

Define the node transfer contribution index as follows:

$$
\varphi_{i}=\frac{\sum_{j \in \Omega} \varphi_{i j}}{D_{i}}
$$

In (8), $\Omega$ is the node set connected to node $\mathrm{i} ; D_{i}$ is the degree value of node $\mathrm{i}$. Its physical meaning is that, $\varphi_{i}$ represents the average contribution that the line connected to node $\mathrm{i}$ makes to node activity; $\sum_{j \in \Omega} \varphi_{i j}$ quantifies the role and activity degree of node $\mathrm{i}$ in power transmission; $D_{i}$ reflects the structural characteristics of nodes in the topology model. Dividing the node activity by node degrees is mainly based on the power or energy angle ${ }^{[18]}$, assuming that the two nodes have the same activity degree. When a node with a large node degree fails or disturbs, the power or energy of the fault or disturbance can be transmitted along the multiple lines which are connected to it, and the impact on the remaining nodes and the line is scattered; However, the transmission channel with a lower number of nodes is single, and the power or energy impact is concentrated, which leads to a large probability of chain fault. The contribution of node transmission reflects the carrying or transmission capacity that the node dealing with the network trend to a certain extent.

According to the concept of PTDF, using the node importance degree evaluation matrix, taking the node energy efficiency value, the importance value of the neighbouring nodes and the value of the node contribution into account, the node flow is defined by

$$
\begin{aligned}
G_{i}^{\prime} & =E_{i} \varphi_{i} \sum_{j=1, j \neq i}^{n} J_{E i j} \\
& =E_{i} \frac{\sum_{j \in \Omega} \varphi_{i j}}{D_{i}} \sum_{j=1, j \neq i}^{n} \frac{\omega_{i j} E_{j}}{K^{2} Z_{i j, e q u}}
\end{aligned}
$$

$G_{E i j}$ indicate the important contribution degree that is the node $\mathrm{j}$ to the node $\mathrm{i}$, and it can be seen from the definition that the value is related to the efficiency and the degree of the node itself. The higher the efficiency value is, the higher the importance contribution to the neighbouring nodes is. 
It can be seen from the above formula that, $G_{i}^{\prime}$ can reflect the bear or transmission capability of a node to the network trend. It not only takes the local characteristics of the node (degree value and adjacent node degree value) and global characteristic (node energy efficiency value) into account, but also considers the physical characteristics of the power grid (line resistance value) and makes up the weak point that the topology model only concerns node topology connection based on electrical coupling strength between nodes. The physical background is more in line with the actual power grid.

In order to better normalize the results, normalized processing $G_{i}^{\prime}\left(G_{\max }^{\prime}, G_{\min }^{\prime}\right.$ are the maximum and the minimum values respectively) can obtain the node flow.

$$
G_{i}=\frac{G_{i}^{\prime}-G_{\min }^{\prime}}{G_{\max }^{\prime}-G_{\min }^{\prime}}
$$

\section{Key node identification process}

The key node identification process of power grid based on node flow is shown in Fig1. The main steps of the method are as follows:

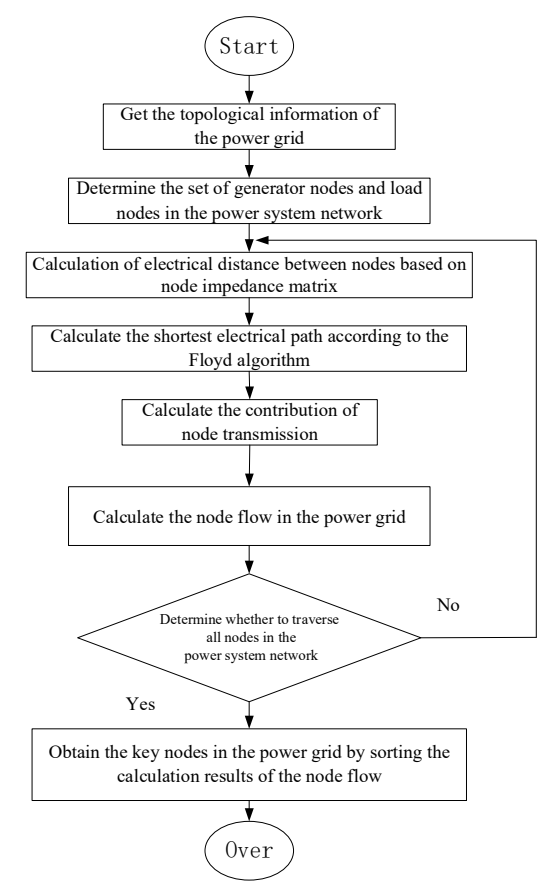

Fig. 1 The flow chart of key nodes identification

1) Get the topological information of the power grid;

2) Determine the set of generator nodes and load nodes in the power system network;

3) According to the node impedance matrix, the value is equal to the voltage between the nodes $\mathrm{g}$ and 1 after injecting the unit current element from the node $\mathrm{g}$. According to the superposition principle, the node impedance matrix element can be used to calculate the electrical distance between nodes;

4) Calculate the shortest electrical path according to the Floyd algorithm;

5) Calculate the contribution of node transmission;

6) Calculate the node flow in the power grid;

7) Determine whether to traverse all nodes in the power grid, if satisfied, turn to step 8), otherwise turn to step 3); 
8) Obtain the key nodes in the power grid by sorting the calculation results of the node flow.

\section{Example analysis}

In order to analyze and verify the correctness and validity of the key node ranking based on the node flow according to the method in the paper, this paper takes 2017 flood season large power flow of a regional power grid to simulate and verified. The PSASP program were used to simulate and analyze. According to the node flow calculation method, all the DC converter station and $500 \mathrm{kV}$ AC substation is calculated, and the first 20 key nodes are obtained, as shown in table 1 . If one node fails, it may cause significant flow transfer, which will lead to cascading failure.

Table 1 The node flow of a regional power grid.

\begin{tabular}{|c|c|c|c|c|}
\hline NO. & Node Type & Node Name & Node Flow & Power through the Node(MW) \\
\hline 1 & converter station & Congxi & 1 & 6400 \\
\hline 2 & converter station & Huidong & 0.834493655 & 5000 \\
\hline 3 & converter station & Qiaoxiang & 0.692758621 & 5000 \\
\hline 4 & substation & Huadu & 0.642068966 & 3800 \\
\hline 5 & substation & Pingguo & 0.546074483 & 3670 \\
\hline 6 & substation & Maoming & 0.405517241 & 2199 \\
\hline 7 & converter station & Zhaoqing & 0.387862069 & 3000 \\
\hline 8 & converter station & Bao’an & 0.358448276 & 3000 \\
\hline 9 & converter station & Guangzhou & 0.287827586 & 1800 \\
\hline 10 & substation & Luodong & 0.279117241 & 4375 \\
\hline 11 & substation & Yulin & 0.253448276 & 3245 \\
\hline 12 & substation & Zengcheng & 0.252563793 & 3506 \\
\hline 13 & substation & Boluo & 0.236551724 & 4816 \\
\hline 14 & substation & Nan'ning & 0.219655172 & 4836 \\
\hline 15 & substation & Laibing & 0.185862069 & 3256 \\
\hline
\end{tabular}

Select Guangzhou converter station (the node flow: 0.287827586, No.9) and Liping substation (the node flow: 0.103448276, No.63) as a comparison, the geographical distribution of the power grid near these two places is shown in Figure 2 and Figure 3. Guangzhou converter station node degree is 3, Liping substation node degree is 4, two nodes are similar degrees; Moreover, Guangzhou converter station is a DC inverter converter station, Liping substation is an important converting station of Guizhou - Guangxi section, a cross has been suitable to be a comparison object.

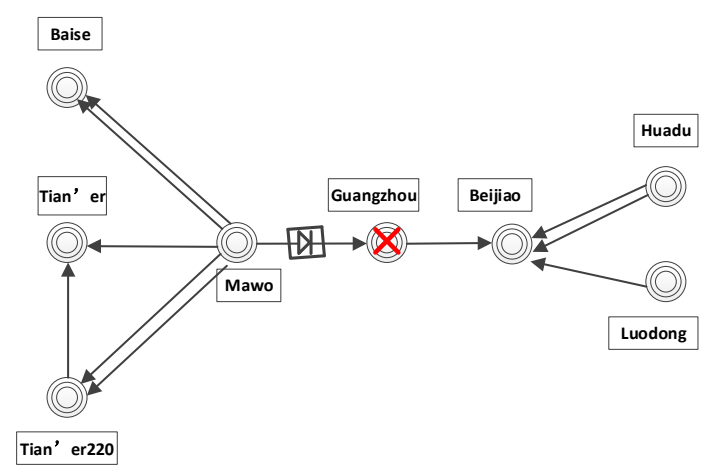

Fig 2 Geographical Connection Diagram of Power Grid in Guangzhou Converter Station 


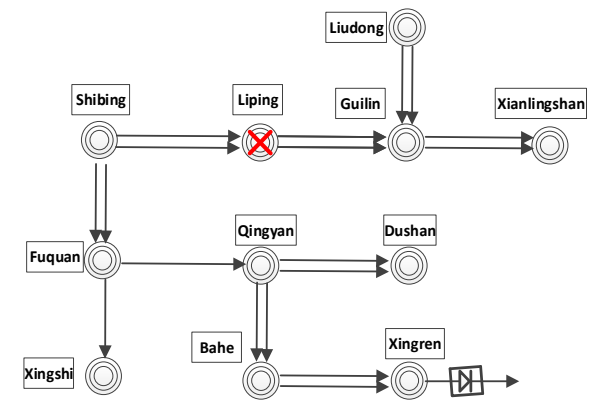

Fig 3 Geographical Connection Diagram of Power Grid in Liping Substation

The lines of Guangzhou and Liping were separate disconnect, and the flow transfer of the AC lines around them was observed. The results are shown in Table 2, Table 3, Figure 4 and Figure 5(assuming a rated load factor of 1 ).

Table 2 The Change of AC Line Load Rate around Guangzhou Converter Station Before and After Remove

\begin{tabular}{|c|c|c|c|}
\hline NO. & Line Name & Load Rate Before Fault & Load Rate After Fault \\
\hline 1 & Mawo - Tian'er220 & 0.142 & 1.453 \\
\hline 2 & Tian'er 220 - Tian'er & 0.045 & 1.131 \\
\hline 3 & Huadu - Beijiao & 0.676 & 0.892 \\
\hline 4 & Beijiao - Luodong & 0.280 & 0.749 \\
\hline 5 & Mawo - Baise & 0.417 & 0.619 \\
\hline 6 & Mawo - Tian'er & 0.116 & 0.438 \\
\hline
\end{tabular}

Table 3 The Change of AC Line Load Rate around Guangzhou Converter Station Before and After Remove

\begin{tabular}{|c|c|c|c|}
\hline NO. & Line Name & Load Rate Before Fault & Load Rate After Fault \\
\hline 1 & Fuquan - Qingyan & 0.189 & 0.624 \\
\hline 2 & Shibing - Fuquan & 0.037 & 0.612 \\
\hline 3 & Liudong - Guilin & 0.253 & 0.609 \\
\hline 4 & Fuquan - Xingshi & 0.110 & 0.461 \\
\hline 5 & Guilin - Xianlingshan & 0.513 & 0.426 \\
\hline 6 & Qingyan - Dushan & 0.178 & 0.379 \\
\hline 7 & Bahe - Xingren & 0.036 & 0.242 \\
\hline
\end{tabular}

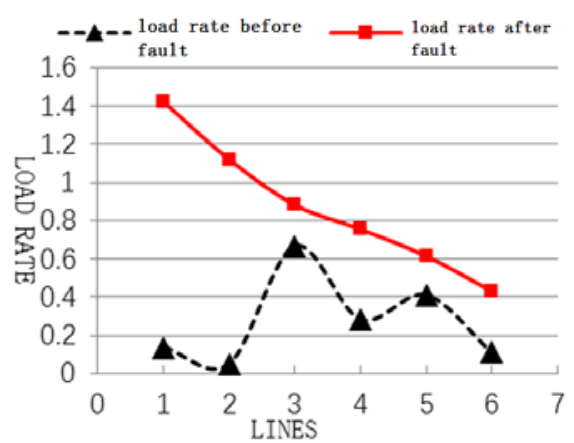

Fig 4 The Change of AC Line Load Rate around Guangzhou Converter Station Before and After Remove 
So, the greater the node flow, the more important the node is in the evolution and propagation of cascading failures. Therefore, the calculation results of the node flow are basically consistent with the simulation results. From the above simulation results, it can also be seen that both from the node degree or power flow, the value of Liping converter station is greater than the value of Guangzhou converter station, but the impact of Liping substation node failure on the power grid is smaller than the impact of Guangzhou converter station caused by the fault. Therefore, identifying the key nodes of the power grid cannot be based solely on node degrees or node power.

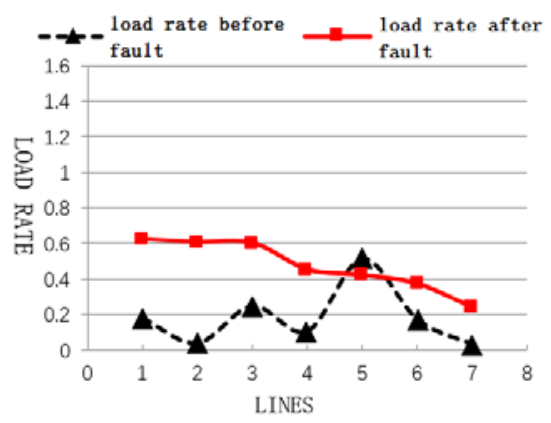

Fig 5 The Change of AC Line Load Rate around Liping Substation Before and After Remove

\section{Conclusion}

In this paper, from the view of node flow, it can quantitatively characterize the decrease of network capacity and the change of network topology before and after node disconnection. In order to characterize the critical degree of the node in the AC/DC hybrid power grid, compared with the network degree in the complex network theory and the network efficiency in the effective performance model, the node flow defined in this paper overcomes the limitation of the conventional method that is only related to the network topology, and uses the change of the network capacity after failure and the electrical coupling strength between nodes to quantitatively characterize the important position of a node in the spread of cascading failure. Therefore, it is of great practical value to establish a more accurate method to identify the key nodes of the AC/DC hybrid power grid, to find the weak lines of the power grid and to prevent the critical state of the fault.

\section{References}

[1] Bompard E,Napoli R,Xue F.Extended topological approach for the assessment of structural vulnerability in transmission networks[J].IET Generation Transmission \& Distribution,2010,4(6):716-724.

[2] TANG Yong,BU Guangquan,YI Jun.Analysis and lessons of the blackout in Indian power grid on July 30 and 31,2012[J].Proceedings of the CSEE,2012,32(35):167-174.

[3] Yin Yonghua,Guo Jianbo,Zhao Jianjun,et al.Preliminary analysis of large scale blackout in interconnected North America power grid on August 14 and lessons to be drawn[J].Power system technology,2003,27(10):8-11.

[4] Hu Xuehao.Rethinking and enlightenment of large scope blackout in interconnected North American power grid[J].Power System Technology,2003,27(9):2-6.

[5] Zhu Guowei,Wang Xianpei,He Ruijuan,et al.Identification of Vital Node in Power Grid Based on Importance Evaluation Matrix[J].High Voltage Engineering,2016,42(10):3347-3353.

[6] $\mathrm{Hu} \mathrm{Yi}, \mathrm{Liu} \mathrm{Kai}, \mathrm{Wu}$ Tian,et al.Analysis of influential factors on operation safety of transmission line and countermeasures[J].High Voltage Engineering,2014,40(11):3491-3499. 
[7] Salmeron J,Wood K,Baldick R.Analysis of electric grid security under terrorist threat[J].IEEE Transactions on Power Systems,2004,19(2):905-912.

[8] Barabasi A L,Albert R.Emergence of scaling in random networks[J].Science, 1999,286(5439):509-512.[9]

[9] Motter A E,Lai Y-C.Cascade-based attacks on complex networks[J].Physical Review E,2002,66(2):1-4.

[10] Kinney R,Crucitti P,Albert R,et al.Modeling cascading failures in the North American power grid[J].The European Physical Journal,2005,46(1):101-107.

[11] Lu Z X,Meng Z W,Zhou S X.Cascading failure analysis of bulk power system using small-world network model[C]//2004 International Conference on Probabilistic Methods Applied to Power Systems.Ames,USA:Iowa State University,2004:635-640.

[12] Cao Yijia,Chen Xiaogang,Sun Ke.Identification of vulnerable lines in power grid based on complex network theory[J].Electric Power Automation Equipment.2006,26(12):1-5,31.

[13] Shi Jin,Tu Guangyu,Luo Yi.Complex network characteristic analysis and model improving of the power system [J].Proceedings of the CSEE,2008,28(25):93-98.

[14] Ju Wenyun,Li Yinhong.Idectification of critical lines and nodes in power grid based on maximum flow transmission contribution degree[J].Automation of Electric Power Systems,2012,36(9):6-12.

[15] Liu Wenying,Liang Cai,Xu Peng,et al.Identification of Critical Line in Power Systems Based on Flow Betweenness [J].Proceedings of the CSEE,2013, 33(31): 90-98.

[16] Wang Tao,Gao Chengbin,Gu Xueping,et al. Power betweenness based identification of power grid critical links[J].Power System Technology, 2014, 38(7): 1907-1913.

[17] LI Yun-kun, Lü Feipeng, JIANG Ke, et al. Method for power system operation mode combination based on shortest electrical distance[J]. Power System Protection and Control, 2010, 38(15): 24-27.

[18] WEI Zhenbo, LIU Junyong, ZHU Mi, et a1. Identification of power topological structure based on network data mining[J]. Automation of Electric Power Systems, 2011, 35(4):12 - 17

[19] Zeng Kaiwen, Wen Jinyu, Cheng Shijie, et al. Critical line identification of complex power system in cascading failure[J]. Proceedings of the CSEE, 2014, 34(7): 1103-1112.

[20] Li Yong,Liu Junyong,Liu Xiaoyu,et al.Vulnerability assessment in power grid cascading failures based on entropy of power flow[J].Automation of Electric Power Systems,2012,36(19):11-16.

[21] Wang Tao,Yue Xianlong,Gu Xueping,et al.Power grid critical node identification based on singular value entropy and power flow distribution entropy[J].Electric Power Automation Equipment,2016,36(4):46-53.

[22] Tan Yudong,Li Xinran,Cai Ye, et al.Critical node identification for complex power grid[J]. Proceedings of the CSEE,2014,34(1):146-152.

[23] Xu Lin,Wang Xiuli,Wang Xifan. Electric betweenness and its application in vulnerable line identification in power system[J]. Proceedings of the CSEE,2010,30(1):33-39.

[24] Su Huiling,Li Yang.Line vulnerability risk analysis based on complex network characteristics of power system[J].Electric Power Automation Equipment,2014,34(2):101-107.

[25] Li Yang,Su Huiling.Critical line affecting power system vulnerability under N-k contingency condition[J].Electric Power Automation Equipment,2015,35(3):60-67.

[26] Zeng Leilei,Yang Qi,Zeng Xin.Identification of Key Vulnerable Lines in AC/DC Hybrid Power Grid[J].Proceedings of the CSU-EPSA,2016,(07):106-111. 